\title{
Reorganization of Mouse Sperm Lipid Rafts by Capacitation
}

\author{
CATHERINE D. THALER,* MONZY THOMAS, AND JENNIFFER R. RAMALIE \\ Department of Biology, University of Central Florida, Orlando, Florida
}

\begin{abstract}
One of the hallmarks of mammalian sperm capacitation is the loss of cholesterol from the plasma membrane. Cholesterol has been associated with the formation of detergent insoluble membrane microdomains in many cell types, and sperm from several mammalian species have been shown to contain detergent-resistant membranes (DRMs). The change in cholesterol composition of the sperm plasma membrane during capacitation raises the question of whether the contents of DRMs are altered during this process. In this study, we investigated changes in protein composition of DRMs isolated from uncapacitated or capacitated mouse sperm. TX-100 insoluble membranes were fractionated by sucrose flotation gradient centrifugation and analyzed by Western and lectin blotting, and capacitation-related differences in protein composition were identified. Following capacitation, the detergent insoluble fractions moved to lighter positions on the sucrose gradients, reflecting a global change in density or composition. We identified several individual proteins that either became enriched or depleted in DRM fractions following capacitation. These data suggest that the physiological changes in sperm motility, ability to penetrate the zona pellucida (ZP), ZP responsiveness, and other capacitation-dependent changes, may be due in part to a functional reorganization of plasma membrane microdomains. Mol. Reprod. Dev. 73: 1541-1549, 2006. (c) 2006 Wiley-Liss, Inc.
\end{abstract}

Key Words: capacitation; lipid raft; membrane organization

\section{INTRODUCTION}

Capacitation represents a series of changes in sperm physiology that are required for sperm to successfully complete fertilization. Uncapacitated sperm are not able to penetrate the cumulus matrix surrounding the oocyte (Talbot, 1985; Corselli and Talbot, 1987), and although they are able to bind to the zona pellucida (ZP), they have a reduced ability to undergo ZP-induced acrosomal exocytosis and a reduced capacity to fertilize in vitro (Visconti et al., 1995a,b). Following capacitation, sperm acquire the capacity to undergo ZP-stimulated acrosomal exocytosis, penetrate the ZP, and fuse with the egg (Cardullo and Thaler, 2002).
A number of molecular changes are associated with capacitation (for review, Jaiswal and Eisenbach, 2002). Protein and lipid distributions on the sperm surface change both during the course of sperm maturation in the epididymis and during capacitation (Saxena et al., 1986; Jones et al., 1990; Cowan et al., 2001; Gadella and Harrison, 2002). In a recent work, detergent-resistant membranes (DRMs) or lipid rafts have been identified in boar (Shadan et al., 2004), and human (Cross, 2004) sperm, as well as mouse (Travis et al., 2001; Sleight et al., 2005) and guinea pig sperm (Travis et al., 2001). Many signaling proteins, both receptors and intracellular mediators of signaling, are associated with DRMs (Foster et al., 2003), indicating that lipid rafts and other membrane microdomains act as carriers for specialized complexes or scaffolds of signal transduction (Hoessli et al., 2000; Foster et al., 2003). These signaling platforms or signalosomes may be used by the cell to integrate information from various signals, or to temporally or spatially regulate signaling (Sowa et al., 2001).

Experimental manipulations such as treatment with $\beta$-methyl-cyclodextrin, which removes cholesterol from membranes, have been shown to disrupt signaling in various systems (Brown and London, 1998) including gametes (Belton et al., 2001). When mammalian sperm undergo capacitation, they lose cholesterol from their plasma membrane (Flesch et al., 2001). This change is required to stimulate cAMP-dependent tyrosine phosphorylation and can be mimicked by addition of $\beta$-methyl-cyclodextrin to a noncapacitating medium (Visconti et al., 1999a). In contrast to other systems, loss of cholesterol from the sperm plasma membrane is correlated with activation of signaling. These changes may be required to assemble the appropriate machinery

Summary Sentence: The protein composition of mouse sperm TX-100 insoluble membrane microdomains changes with capacitation.

Grant sponsor: NIH; Grant number: HD38125.

Monzy Thomas's present address is Department of Pathology, University of Michigan, M4233 Medical Science I 0602, Ann Arbor, MI 48109.

*Correspondence to: Catherine D. Thaler, Hopkins Marine Station, Stanford University, 120 Ocean View Blvd., Pacific Grove, CA 93950. E-mail: cthaler@stanford.edu

Received 8 February 2006; Accepted 20 March 2006

Published online 8 August 2006 in Wiley InterScience

(www.interscience.wiley.com).

DOI $10.1002 / \operatorname{mrd} .20540$ 
to stimulate tyrosine phosphorylation (Visconti et al., $1999 \mathrm{a}, \mathrm{b})$, or the ZP-induced acrosome reaction (Ward and Kopf, 1993).

Previously, we have shown that populations of capacitated or uncapacitated mouse sperm displayed significantly different patterns of lectin staining on the sperm head (Baker et al., 2004). In this study, we analyzed the composition of TX-100 insoluble membrane fractions from uncapacitated or capacitated sperm. Following capacitation, a number of proteins identified by Western or lectin blotting show an altered distribution among the DRM fractions isolated from sucrose flotation gradients. Additionally, only some of the DRM fractions contain caveolin-1, suggesting that at least two types of DRMs are present in mouse sperm, caveolin-1 containing microdomains and noncaveolin, or lipid raft, domains (Brown and Jacobson, 2001).

\section{MATERIALS AND METHODS Materials}

Antibodies were purchased from Zymed, South San Francisco, CA (anti-phosphotyrosine, PY-20), Transduction Laboratories, Lexington, KY (anti-caveolin-1), Sigma, St. Louis, MO (anti- $\beta$-tubulin), Promega, Madison, WI (alkaline phosphatase-conjugated goatanti-rabbit), and Chemicon, Temecula, CA (alkaline phosphatase-conjugated affinity purified goat-antimouse). Biotinylated lectins were purchased from EY-Laboratories (San Mateo, CA) or Sigma. Avidinconjugated alkaline phosphatase was purchased from Vector Labs (Burlingame, CA). Electrophoresis and blotting materials were purchased from Bio-Rad (Hercules, CA). Mice were purchased from HarlanSprague-Dawley (Indianapolis, IN). BSA (fraction V), fetal bovine serum, sodium lactate, sodium pyruvate, and sodium bicarbonate were obtained from Sigma. All other chemicals were purchased from Fisher Scientific (Pittsburgh, PA).

\section{Gamete Collection and Handling}

All animal care, handling and euthanasia procedures were approved by the Institutional Animal Care and Use Committee. Male ICR mice, 12-15 weeks of age (Harlan Sprague Dawley) were sacrificed and the cauda epididymides removed. Uncapacitated sperm were collected by mincing the epididymides in M2 salts $(94.66 \mathrm{mM}$ $\mathrm{NaCl}, 4.78 \mathrm{mM} \mathrm{KCl}, 1.19 \mathrm{mM} \mathrm{KH}_{2} \mathrm{PO}_{4}, 1.19 \mathrm{mM} \mathrm{MgSO}_{4}$, $20.85 \mathrm{mM}$ Hepes, $\mathrm{pH}$ 7.4) and allowing sperm to swim out for $20 \mathrm{~min}$ at $37^{\circ} \mathrm{C}$. Tissue debris was removed by centrifugation $(20 \mathrm{~g}, 1 \mathrm{~min}$ ) and the sperm suspension collected and processed for membrane collection as described below. For capacitated sperm preparations, epididymides were minced in M2 buffer (M2 salts plus $23.28 \mathrm{mM}$ Na-Lactate, $5.56 \mathrm{mM}$ Glucose, $4.15 \mathrm{mM}$ $\mathrm{NaHCO}_{3}, 0.5 \mathrm{mM} \mathrm{Ca}^{2+}, 0.33 \mathrm{mM}$ Na-Pyruvate, $4 \mathrm{mg} / \mathrm{ml}$ $\mathrm{BSA}, \mathrm{pH}$ 7.4) and incubated at $37^{\circ} \mathrm{C}, 5 \% \mathrm{CO}_{2}$, in a humidified atmosphere for $1 \mathrm{hr}$. Tissue debris was removed by centrifugation $(20 \mathrm{~g}, 1 \mathrm{~min})$. The sperm suspension was collected and membranes prepared, as described below.

\section{Membrane Preparation}

Sperm membranes were isolated as previously described (Baker et al., 2002). Briefly, suspensions of either uncapacitated or capacitated sperm were pelleted $(1,000 \mathrm{~g}, 15 \mathrm{~min})$ and supernatant removed. Sperm were washed in M2 salts and pelleted. The sperm pellet was resuspended in hypotonic buffer ( $2 \mathrm{mM}$ Tris, $\mathrm{pH} 7.2$, $12 \mathrm{mM} \mathrm{NaCl})$ with protease inhibitors $(10 \mu \mathrm{g} / \mathrm{ml}$ aprotinin, $10 \mu \mathrm{g} / \mathrm{ml}$ leupeptin, $1 \mathrm{mM}$ PMSF). The sperm suspension was sonicated four times for 15 sec on ice, with each pulse separated by $1 \mathrm{~min}$. Following a lowspeed spin $(2,500 \mathrm{~g}, 15 \mathrm{~min})$ to remove cell debris, the supernatant containing crude sperm membranes was collected and centrifuged for $60 \mathrm{~min}$ at $108,000 \mathrm{~g}, 4^{\circ} \mathrm{C}$. The membrane pellet was resuspended in buffer $(20 \mathrm{mM}$ Tris, $\mathrm{pH} 7.2,120 \mathrm{mM} \mathrm{NaCl}$ ) and protease inhibitors, as above, by brief sonication. Membranes were frozen in liquid nitrogen and stored under liquid nitrogen until used for preparation of DRMs.

\section{Preparation of TX-100 Insoluble Membranes}

Flotation gradients were prepared with minor modifications of a previously reported procedure (Chamberlain et al., 2001), using crude sperm membranes from 45 mice for each gradient. Briefly, solutions of 2\% TX-100 in MES-buffered saline (MBS: $17 \mathrm{mM}$ MES, pH 6.5, $150 \mathrm{mM} \mathrm{NaCl}$ ) were equilibrated to $4^{\circ} \mathrm{C}$. Crude membranes from capacitated or uncapacitated sperm were sonicated briefly to disrupt aggregations that formed when frozen. Membrane suspensions were placed in an ice water bath to cool and then an equal volume of cold $2 \%$ TX-100 buffer was added and mixed by inverting the tubes. Tubes were held on ice for $10 \mathrm{~min}$. The TX-100 membrane preparations were adjusted to $40 \%$ sucrose by mixing with an equal volume of $80 \%$ sucrose and $1 \mathrm{ml}$ was layered on the bottom of a centrifuge tube. The $40 \%$ sucrose/membrane preparation was overlaid successively with $3 \mathrm{ml}$ of $30 \%$ sucrose and $1 \mathrm{ml}$ of $5 \%$ sucrose. The flotation gradients were centrifuged at $250,000 \mathrm{~g}, 4^{\circ} \mathrm{C}$, for $19 \mathrm{hr}$ (Beckman SW 50.1ti). Membrane components were visible near the $5 / 30$ interface in both uncapacitated and capacitated sperm membrane preparations. Ten fractions of $500 \mu \mathrm{l}$ each were collected from the top of the gradient and designated fraction 1 (top) through 10 (bottom). A pellet fraction was also collected. The fractions were diluted to $5 \mathrm{ml}$ with MBS and centrifuged at $108,000 \mathrm{~g}$ for $60 \mathrm{~min}$ to pellet membrane components. The supernatant was removed and the pelleted material was prepared for electrophoresis. Two independently prepared gradients for uncapacitated DRMs and two for capacitated DRMs were analyzed in this study.

\section{Electrophoresis and Blotting}

Pellets of the sucrose gradient fractions were solubilized in SDS-PAGE reducing sample buffer, boiled for $5 \mathrm{~min}$, and the entire sample loaded onto $10 \%$ SDSPAGE gels and separated by electrophoresis. Proteins were transferred to nitrocellulose (Otter et al., 1988) and 
the blots were stored at $-20^{\circ} \mathrm{C}$ until probed. Blots of the membrane fractions were probed with antibodies or lectins. The antibodies used in this study were: antiphosphotyrosine (PY-20), anti-caveolin 1 , anti- $\beta$-tubulin, and anti-actin. The lectins used in this study were: Arachis hypogaea (PNA), Anguilla anguilla (AAA), Lycopersicon esculentum (LEA), Pisum sativum (PSA), Solanum tuberosum (STA), Tetragonolobus purpureas (TPA), Ulex europaeus I (UEA-1), and Triticum vulgare (WGA).

Antibody staining. Blots were blocked overnight in TBS, $4 \%$ BSA, $1 \%$ Ovalbumin, $0.1 \%$ Tween-20, for anticaveolin1 and anti-phosphotyrosine, or TBS, 5\% nonfat dry milk, $0.2 \%$ Tween- 20 for anti- $\beta$-tubulin and antiactin. The blots were incubated with primary antibody in blocking solution for $1 \mathrm{hr}$, washed thoroughly in blocking solution, incubated with alkaline phosphataseconjugated secondary antibody for $1 \mathrm{hr}$, washed thoroughly, and reactive proteins detected using the Lumiphos $^{\mathrm{TM}}$ substrate (Pierce Biotechnology, Inc., Rockford, IL) and Classic Blue BX autoradiography film (Midwest Scientific, St. Louis, MO).

Lectin staining. Blots were blocked $2 \mathrm{hr}$ at room temperature, or overnight a $4^{\circ} \mathrm{C}$, in TBS with $3 \% \mathrm{BSA}$, $0.2 \%$ Tween-20. The blots were incubated with biotinylated lectins for $2 \mathrm{hr}$, washed extensively in TBS, $0.2 \%$ Tween, $1 \%$ BSA, incubated with alkaline phosphataseconjugated avidin $(1 \mu \mathrm{g} / \mathrm{ml})$ for $1 \mathrm{hr}$, and then washed thoroughly. Lectin reactive glycoproteins were detected using the Lumiphos ${ }^{\mathrm{TM}}$ substrate (Pierce) and Classic Blue BX film (Midwest).

Reprobing. Blots were stripped with $62.5 \mathrm{mM}$ Tris$\mathrm{HCl}, \mathrm{pH} 6.7,100 \mathrm{mM} \beta$-mercaptoethanol, 2\% SDS, at $50^{\circ} \mathrm{C}$ for $30 \mathrm{~min}$ and washed thoroughly with TBS. Blots were then blocked and probed with additional antibodies or lectins.

Densitometry. For quantitation of proteins, images of the Western or lectin blots were captured from film and area densities of particular bands were determined using Labworks ${ }^{\mathrm{TM}}$ software (UVP, Inc., Upland, CA). Area density calculations were performed for each antibody or lectin staining band of interest using a uniformly sized region of interest. Area densities were normalized to percent of total reactivity, as follows:

$$
\begin{aligned}
& \text { \% Total Distribution } \\
& \quad=\left[\frac{\text { Density of Band X in Fraction Y }}{\left.\sum \text { (Density of Band X in all Fractions }\right)}\right] \times 100
\end{aligned}
$$

Comparisons between uncapacitated and capacitated samples were made using \% Total Distribution.

\section{RESULTS}

\section{Experimental Rationale}

Crude sperm membranes, prepared by sonication, from sperm that were incubated in a capacitating medium for $1 \mathrm{hr}$, or from sperm collected in a noncapacitating medium, were used as a starting material for isolation of DRMs. We have previously shown that sonication is able to remove greater than $90 \%$ of membranes from mouse sperm (Baker et al., 2002). DRMs were prepared from suspensions of crude sperm membranes and fractionated by flotation on a sucrose step gradient. In order to make relevant comparisons between uncapacitated and capacitated samples, the methods for preparation of the DRMs were identical for uncapacitated and capacitated samples. A number of different types of detergent insoluble membrane microdomains have been described in the literature (Brown and Jacobson, 2001; Chamberlain, 2004) and a number of fine molecular distinctions have arisen. In this initial study we have used the general term DRMs to describe membrane fractions which were both: (1) insoluble in ice-cold 1\% TX-100, and (2) found near the $5-30 \%$ interface of a sucrose flotation gradient after centrifugation. Insoluble material was recovered from each fraction of the flotation gradient by ultracentrifugation and analyzed by immuno- and lectin-blotting to identify changes in protein composition and/or phosphorylation state. DRMs were found in Fractions \#2-7 and these fractions were used for quantitative comparisons.

\section{Both Uncapacitated and Capacitated Sperm Possess DRMs}

Flotation gradients prepared from uncapacitated or capacitated crude sperm membranes contained DRM fractions detected by visual inspection in Fractions \#3-5 (data not shown) and by SDS-PAGE in Fractions \#2-7 (Fig. 1). Flotation gradient fractions probed with the anti-phosphotyrosine antibody PY-20, revealed the presence of DRMs in both uncapacitated and capacitated samples (Fig. 1A). In the uncapacitated samples, the major tyrosine phosphorylated protein of mouse sperm, p116/hexokinase (Visconti et al., 1996), appeared strongly in the light membrane fractions (Fractions \#4-6) but was also present in the heavier membranes of Fraction 10 and in the pellet. In the capacitated sperm DRMs, the majority of the p116/hexokinase shifted to lighter fractions of the gradient, appearing as high as Fraction \#2. The most intensely stained Fractions, \#3 and 4, also contained several other tyrosine phosphorylated proteins, indicative of capacitated sperm (Visconti et al., 1995a,b). Overall, the amount of p116 in the light membrane fractions (Fractions \#2-7) is similar in uncapacitated $(69.3 \%)$ versus capacitated $(73.5 \%)$ preparations, but the relative position has shifted toward lighter fractions of the gradient.

The distribution of the hexokinase was mirrored by caveolin-1 (Fig. 1B), which was also detected in the DRM fractions and shifted to lighter density fractions in the capacitated sperm samples. In both uncapacitated and capacitated samples, some detergent-resistant components were found in fractions that did not contain caveolin, Fraction \#4 in uncapacitated sperm membranes and Fraction \#2 in capacitated sperm membranes (compare Fig. 1A,B). In uncapacitated samples, $100 \%$ of the caveolin detected was in the DRM fractions, but only $86.5 \%$ was found in the DRMs of capacitated samples. 
A
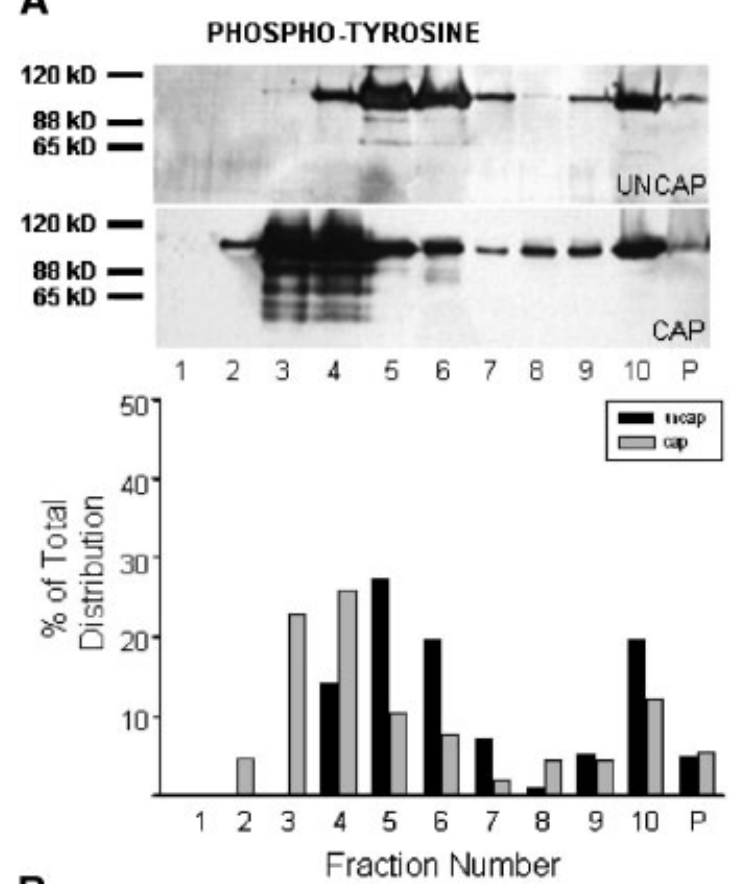

B

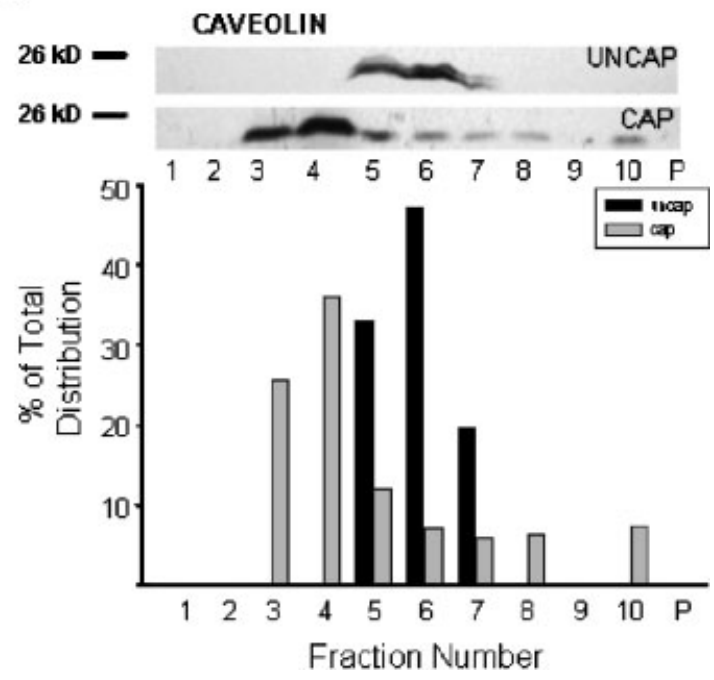

Fig. 1. Protein association with detergent-resistant membranes (DRMs) changes with capacitation state. A: Phosphotyrosine containing proteins were identified with the PY-20 antibody. The major phosphoprotein of uncapacitated sperm is the p116/hexokinase, and a significant fraction is found in DRMs. In capacitated sperm membranes, p116 shifts into lighter fractions, and additional tyrosine phosphorylated proteins which are capacitation specific appear in these fractions. The distribution histogram quantifies the fraction of p116/hexokinase in each of the gradient fractions. B: Caveolin-1 distribution, before and after capacitation, mirrors the p116 distribution, being primarily in DRMs and moving to lighter fractions with capacitation.

In contrast, $\beta$-tubulin remained exclusively in the heavy fractions and pellet (Fig. 2A). Actin, however, was found in the heavy fractions as well as DRM fractions of uncapacitated sperm, but moved almost completely to the heavy fractions in capacitated sperm (Fig. 2B). In uncapacitated samples, $25.7 \%$ of the actin was asso-
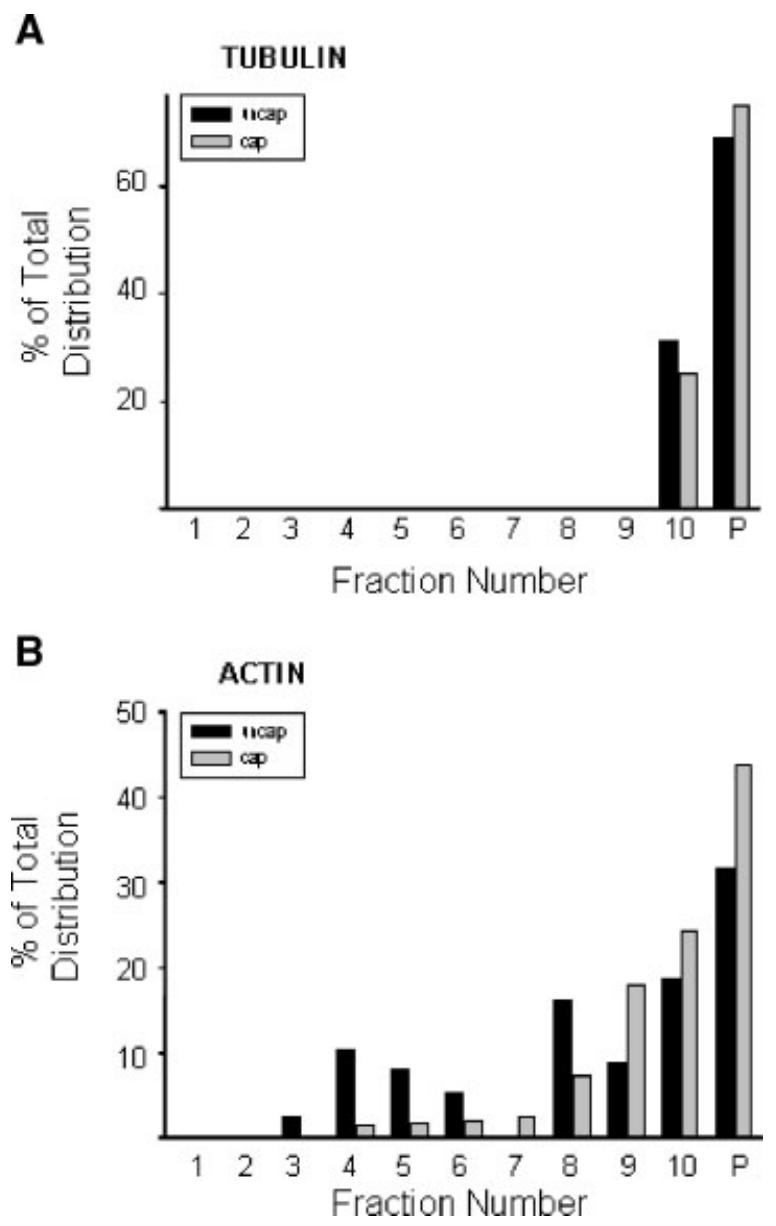

Fig. 2. Actin, but not tubulin, associates with DRMs. A: $\beta$-tubulin did not associate with DRMs and was found exclusively in the lower fractions of the gradient in both uncapacitated and capacitated sperm DRMs. B: Actin was detected in the DRMs of uncapacitated sperm membranes, but shifted to the heavier fractions with capacitation.

ciated with the DRM fractions, but only $7.4 \%$ was found in DRMs of capacitated samples, a 3.2-fold difference.

\section{Lectin Reactive Proteins Are Differentially Localized Depending on Capacitation State}

Formation and reorganization of protein domains on the sperm head has been noted in many mammalian species (e.g., Peterson and Russell, 1985; Saxena et al., 1986; Bearer and Friend, 1990; Jones et al., 1990; Cardullo and Wolf, 1995; Cowan et al., 2001). In a previous study, we found that capacitation-associated protein reorganizations of the mouse sperm head could be followed by lectin fluorescence (Baker et al., 2004). Lectin blotting of DRMs from uncapacitated and capacitated sperm membrane samples enabled us to identify specific plasma membrane components associated with DRMs. Lectin reactive bands were found in both DRMs and heavy membrane fractions and many of these bands did not change distribution with capacitation (data not shown). However, a number of bands whose association with the DRMs changed as a result of capacitation were identified, including, for example, a 

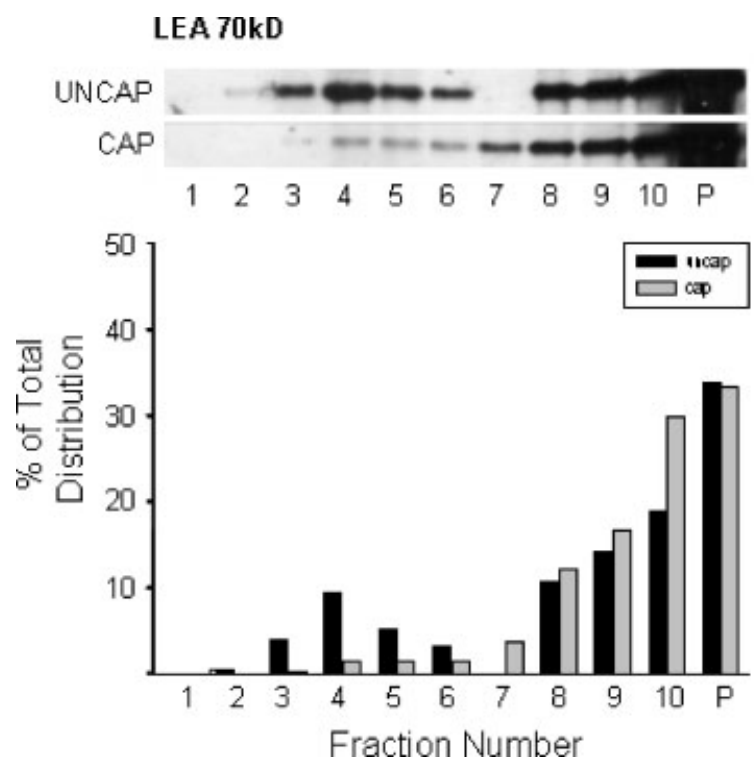

Fig. 3. LEA-70 leaves the DRMs of capacitated sperm. A $70 \mathrm{kDa}$ LEA reactive polypeptide localized to DRM fractions in uncapacitated sperm, but moved into the heavier fractions in capacitated sperm preparations.

$70 \mathrm{kDa}$ LEA reactive band (Fig. 3). A fraction of LEA-70 is associated with DRMs of uncapacitated sperm (27\%), but leaves the DRM fractions in capacitated sperm, with only $9.9 \%$ of the protein remaining with the DRMs, a 2.7 fold change. This is similar to the behavior of actin, but contrasts the behavior of p116/hexokinasse and caveolin-1, both of which remained in light membrane fractions and shifted position to lighter fractions with capacitation.

We identified three trends in DRM-associated protein behavior relative to capacitation state, as illustrated in Figure 4. TREND 1: the protein shows no change in DRM association relative to capacitation. PSA-30, for example, remained associated with the DRM fractions (95.9\% in uncapacitated preparations vs. $91.0 \%$ in capacitated preparations (Fig. 4A)). However, the distribution of PSA-30 shifted to lighter fractions, Fractions \#3 and 4 in capacitated sperm versus Fractions \#5 and 6 in uncapacitated sperm. TREND 2: the protein enters the DRM fractions upon capacitation. STA-26, for example, displayed a net increase in protein associated with DRM fractions (69.8\% in uncapacitated sperm, $92.1 \%$ in capacitated sperm), and the majority of STA-26 moved into the lighter fractions seen only in capacitated preparations (Fig. 4B). TREND 3: the protein leaves the DRM fractions upon capacitation. PSA-70, for example, left the DRM fractions under capacitating conditions (84.0\% in uncapacitated sperm membranes, $36.2 \%$ in capacitated (Fig. 4C)), similar to the behavior of LEA-70 (Fig. 3). Although the amount of PSA-70 associated with DRMs was considerably reduced, the fraction that stayed with the DRMs moved into the lighter, capacitation-related fractions. Two lectins, PNA and UEA-1, bind components on the mouse sperm head, as shown by lectin fluorescence [23], but did not detect any glycopro-
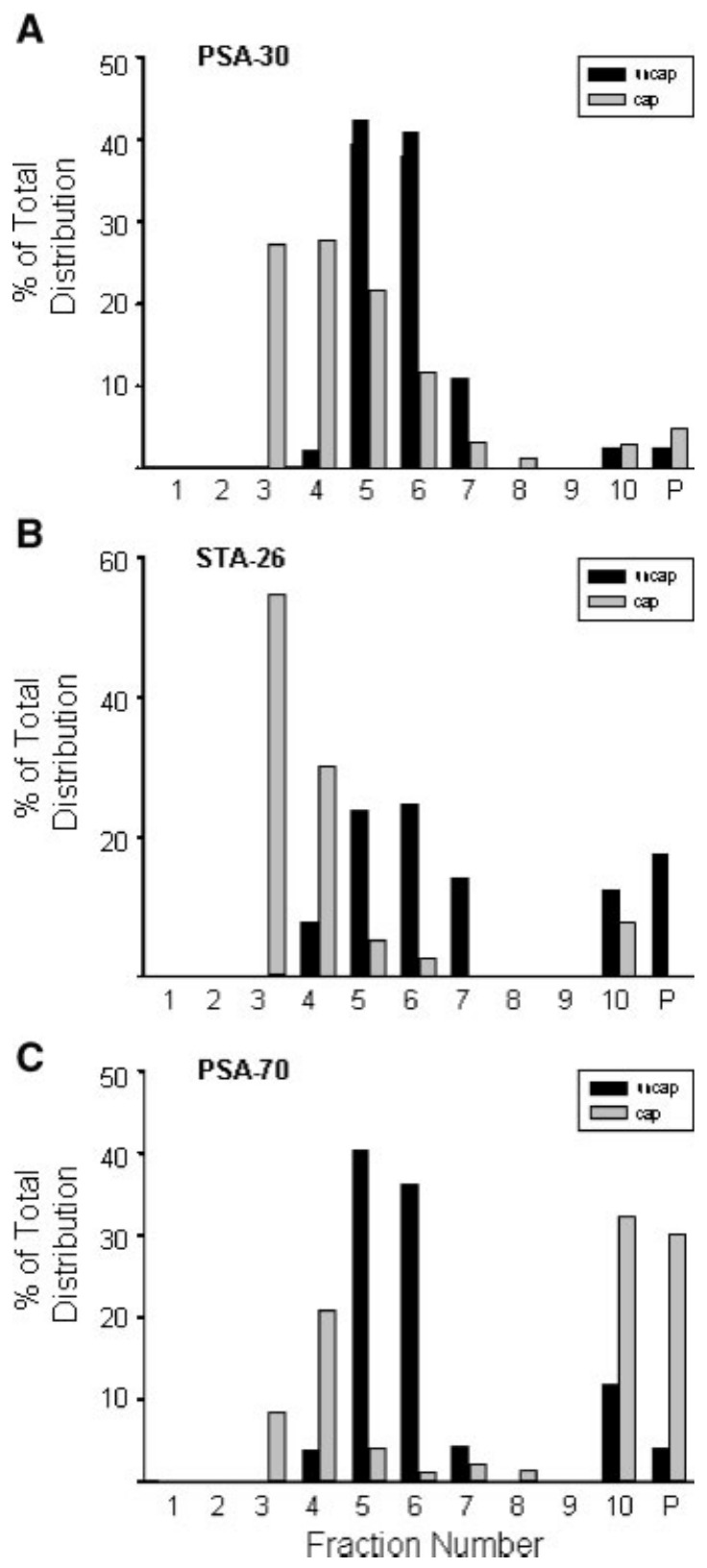

Fig. 4. Sperm membrane glycoproteins show variable behavior in redistribution during capacitation. Three capacitation-associated trends are exemplified by the following proteins. A: PSA-30. There is no change in the amount of PSA-30 associated with DRMs in uncapacitated and capacitated sperm, but the majority of PSA-30 localizes to lighter fractions of the gradient in the capacitated sperm membranes. B: STA-26. STA-26 enters the DRM fractions upon capacitation, and more is found in the lighter fractions. C: PSA-70. PSA-70 leaves the DRM fractions as a result of capacitation.

teins when used in blotting of the sucrose flotation gradient fractions (data not shown).

We quantified prominent bands stained by each of the lectins to illustrate the changes in membrane distribution correlated with capacitation (Table 1). Most of the lectins used in this study stained several DRMassociated polypeptides, many of which were associated with DRMs in both uncapacitated and capacitated 
Molecular Reproduction and Development. DOI 10.1002/mrd

\section{C.D. THALER ET AL.}

TABLE 1. Protein Redistributions in TX-100 Insoluble Membrane Fractions Are Correlated With Capacitation

\begin{tabular}{lccl}
\hline & \multicolumn{2}{c}{$\%$ Protein in light membrane fractions ${ }^{\mathrm{b}}$} & \\
\cline { 2 - 3 } Polypeptide $^{\mathrm{a}}$ & Uncapacitated & Capacitated & \multicolumn{1}{c}{ Trend $^{\mathrm{c}}$} \\
\hline p116 & 69.3 & 73.5 & No change \\
caveolin-1 & 100 & 86.5 & Leaving \\
$\beta$-tubulin & 0 & 0 & Not DRM associated \\
actin & 25.7 & 7.4 & Leaving \\
LEA-70 & 27.0 & 9.9 & Leaving \\
LEA-30 & 96.4 & 80.0 & Leaving \\
PSA-70 & 84.0 & 36.3 & Leaving \\
PSA-30 & 95.9 & 91.0 & No change \\
STA-116 & 55.9 & 73.4 & Entering \\
STA-26 & 69.8 & 92.1 & Entering \\
WGA-100 & 67.3 & 80.9 & Entering \\
WGA-40 & 58.3 & 66.1 & No change \\
AAA-32 & 43.7 & 39.3 & No change \\
AAA-25 & 89.1 & 71.5 & Leaving \\
TPA-60 & 0 & 0 & Not DRM associated \\
\hline
\end{tabular}

${ }^{a}$ Lectin reactive glycoproteins are identified by the lectin and their apparent molecular mass in $\mathrm{kDa}$.

${ }^{b}$ The amount of each protein in Fractions \#2-7 of the flotation gradient, as a percentage of the total amount of that protein detected in the gradient. Values are the average of density measurements from two independent gradients for each condition (capacitated or uncapacitated).

'The trend in distribution of a protein in the flotation gradient fractions, relative to capacitation of the sperm. Changes in relative amounts of protein in the light membrane Fractions (\#2-7) are considered reflective of the association with DRMs, and are characterized as entering or leaving the DRM fractions, no change, or not DRM associated. Proteins in the DRM fractions shifted to higher (lighter) fractions in the gradient in capacitated sperm samples, even if the net trend was the antigen leaving the DRM fractions. See PSA-70 (Fig. 4), for example.

membranes (TREND 1), but a number of lectin staining polypeptides did change their distribution relative to capacitation state (TREND 2 and 3). An interesting exception is the major protein recognized by the TPA lectin, TPA-60. TPA-60 was found exclusively in the denser fractions of the gradient and did not change with capacitation state (Table 1), consistent with our previous findings that fluorescent staining with TPA revealed identical patterns in uncapacitated or capacitated sperm (Baker et al., 2004).

\section{DISCUSSION}

In this study, we used sucrose flotation gradient fractionation of TX-100 DRMs to compare the organization of uncapacitated and capacitated mouse sperm plasma membranes. We identified two types of DRMs, caveolin-1 containing light membranes, and those in which caveolin-1 was absent. Changes in both the physical properties (flotation on a sucrose gradient) and the molecular components (plasma membrane proteins) were observed. Capacitation results in an overall shift in the density of DRMs on the flotation gradient. Capacitated sperm DRMs band at a lighter density in the gradient, shifting from a peak at fractions $5 / 6$ for uncapacitated sperm membranes to a peak at fractions $3 / 4$ in capacitated sperm. This corresponded to a similar shift in the position of peak fractions of caveolin-1.

Changes in protein composition of the light membrane fractions were identified by Western and lectin blotting analysis of membrane fractions from uncapacitated or capacitated sperm. Uncapacitated sperm possess a DRM fraction which contains the major tyrosine phosphorylated sperm protein, the p116 hexokinase, and caveolin-1, as previously reported (Travis et al., 2001). Capacitation results in tyrosine phosphorylation of several additional proteins (Visconti et al., 1995a) and these proteins were localized to the DRM fractions in this study. The presence of a suite of tyrosine phosphorylated proteins $(45-90 \mathrm{kDa})$ in the membrane samples from capacitated sperm confirms that the in vitro incubation conditions induced the molecular phenotype of capacitation (Visconti et al., 1995a). Using lectin staining to monitor changes in plasma membrane composition during sperm capacitation, we recently showed that the staining pattern of several lectins displayed significantly different patterns on the sperm head of uncapacitated versus capacitated mouse sperm (Baker et al., 2004). In this study, we detected several lectin reactive glycoproteins whose association with DRM fractions was altered as a result of capacitation. Travis et al. (2001) showed that caveolin-1 was present in the head and tail of both guinea pig and mouse sperm; but our previous study (Baker et al., 2004), did not detect changes in the lectin distribution of tail glycoproteins, suggesting that most plasma membrane reorganizations take place in the head.

Several studies have identified sperm surface antigens whose localization on the sperm head changes during sperm maturation and capacitation (Peterson 
and Russell, 1985; Saxena et al., 1986; Bearer and Friend, 1990; Jones et al., 1990; Cardullo and Wolf, 1995; Cowan et al., 2001). In the current study, we show that the composition of DRMs in the mouse sperm plasma membrane is substantially modified during capacitation. This is the first report to correlate changes in surface distribution of particular protein antigens with changes in DRM association in mouse sperm. Plasma membrane proteins identified by their lectin binding properties were seen to both change location on the sperm head (Baker et al., 2004) and to change position on a sucrose flotation gradient used to isolate DRMs (this study).

Previous work has documented the existence of lipid rafts in mammalian sperm from several species (Travis et al., 2001; Trevino et al., 2001; Cross, 2004; Shadan et al., 2004; Sleight et al., 2005; van Gestel et al., 2005; Selvaraj et al., 2006), and the most recent studies have identified changes in the composition of lipid rafts in mouse, boar, and human sperm. Shadan et al. (2004) showed capacitation-associated changes in the distribution of GM-1 in boar and mouse sperm. Cross (2004) examined flotillin-2, GM-1, and CD59 distributions in human sperm and identified two distinct types of DRMs, as judged by the distinct localization of GM-1 and CD59, similar to our observations here that mouse sperm contains caveolin and noncaveolin containing detergent insoluble membranes. Additionally, consistent with the behavior of several antigens observed here, the fraction of raft-associated GM-1 and CD59 decreased as a result of capacitation. In contrast, Sleight et al. (2005) found an overall decrease in the occurrence of lipid rafts in capacitated mouse sperm. Additionally, the changes in distribution of the $\mathrm{p} 116 /$ hexokinase were different than those reported here. Although the capacitation times and medium are similar in the two studies, the detergent (0.5\% TX-110 in Sleight et al., 2005 vs. $1 \%$ in this study) and buffers (TEN, pH 7.3, in Sleight et al., 2005 , vs. MBS, $\mathrm{pH} 6.5$, in this study) used for isolation of DRMs were different. These differences may account for the conflicting results between the two studies, as many reports have pointed out that different conditions for DRM preparation yield significantly different behaviors of lipid microdomains (see Chamberlain, 2004, for review).

Actin was observed to move out of the DRM fractions in capacitated sperm. Actin has been localized to the acrosomal crescent and tail in mouse sperm (Maier et al., 2003) and capacitation-related changes in the actin cytoskeleton have recently been characterized in sperm from rat, boar, mouse, human (Brener et al., 2003), and guinea pig (Cabello-Agueros et al., 2003; DelgadoBuenrostro et al., 2005). Actin polymerization in bovine sperm was induced by capacitating treatments (Brener et al., 2003) and these data suggest that actin polymerization and changes in filament structure or orientation during capacitation could be responsible for the loss of association with DRMs.

One of the major features of capacitation is that the sperm plasma membrane loses cholesterol. In fact, cholesterol extracting drugs like $\beta$-methyl-cyclodextrin promote capacitation-like changes in an otherwise noncapacitating medium (Visconti et al., 1999a,b; Shadan et al., 2004). Cholesterol content of the plasma membrane is correlated with the presence of lipid rafts, and lipid rafts, in turn, have been intimately correlated with cell signaling. Extraction of cholesterol from the plasma membrane can lead to disruption of normal signaling (Salaun et al., 2004). Cholesterol depletion of PC12 cells, for example, was shown to significantly decrease regulated secretion (Chamberlain et al., 2001). Alternatively, in some cases, release of a protein from the raft environment (Prior et al., 2001), or from association with caveolin-1 (Sowa et al., 2001), has been shown to promote signaling. Thus, normal signaling pathways may be spatially and temporally regulated by the ability of signaling components to associate with lipid rafts (Golub et al., 2004).

Sperm motility, ability to penetrate the cumulus matrix, and ability to undergo ZP-stimulated acrosome reactions all change as a result of capacitation. These and other changes in sperm physiology could result from changes in the composition and localization of membrane domains. A number of studies have documented differences in association of signaling components with caveolae versus lipid rafts (Sowa et al., 2001; Fielding and Fielding, 2004), and such differences may mediate spatial and temporal regulation of signal transduction. Many proteins have been shown to associate preferentially with one type of microdomain even in cells that may contain multiple types of membrane microdomains (Schnitzer et al., 1995b; Madore et al., 1999; Oh and Schnitzer, 2001). Additionally, proteins mediating exocytosis, are targeted to lipid rafts (Schnitzer et al., 1995a; Chamberlain et al., 2001; Travis et al., 2001; Salaun et al., 2004, 2005), suggesting that cells may use membrane microdomains to exert spatial control over sites of exocytosis. These data are consistent with the observations that uncapacitated sperm are refractory to ZP-induced acrosomal exocytosis, but become sensitive to ZP signals following capacitation.

Thus, the capacitation-associated reorganization of membrane microdomains may play a key role in responsiveness to $\mathrm{ZP}$ signaling of acrosomal exocytosis. The changing interactions among plasma membrane components as a result of cholesterol extraction during capacitation may enable not only the intracellular changes associated with capacitation (e.g., tyrosine phosphorylation), but also assembly of surface components to form a signaling platform (Hoessli et al., 2000) for ZP binding and acrosomal exocytosis, and possibly sperm egg interactions at the egg plasma membrane as well.

\section{ACKNOWLEDGMENTS}

This work was supported by NIH HD38125 (C.D.T).

\section{REFERENCES}

Baker SS, Cardullo RA, Thaler CD. 2002. Sonication of mouse sperm membranes reveals distinct protein domains. Biol Reprod 66:57-64. 


\section{C.D. THALER ET AL.}

Baker SS, Thomas M, Thaler CD. 2004. Sperm membrane dynamics assessed by changes in lectin fluorescence before and after capacitation. J Androl 25:744-751.

Bearer EL, Friend DS. 1990. Morphology of mammalian sperm membranes during differentiation, maturation, and capacitation. J Electron Microsc Tech 16:281-297.

Belton RJ, Jr., Adams NL, Foltz KR. 2001. Isolation and characterization of sea urchin egg lipid rafts and their possible function during fertilization. Mol Reprod Dev 59:294-305.

Brener E, Rubinstein S, Cohen G, Shternall K, Rivlin J, Breitbart H. 2003. Remodeling of the actin cytoskeleton during mammalian sperm capacitation and acrosome reaction. Biol Reprod 68:837-845.

Brown DA, Jacobson K. 2001. Microdomains, lipid rafts and caveolae. Traffic 2:668-672.

Brown DA, London E. 1998. Functions of lipid rafts in biological membranes. Annu Rev Cell Dev Biol 14:111-136.

Cabello-Agueros JF, Hernandez-Gonzalez EO, Mujica A. 2003. The role of $\mathrm{F}$-actin cytoskeleton-associated gelsolin in the guinea pig capacitation and acrosome reaction. Cell Motil Cytoskeleton 56:94108.

Cardullo RA, Thaler CD. 2002. Function of the egg's extracellular matrix. In: Hardy DM, editor. Fertilization. San Diego: Academic Press. pp 119-152.

Cardullo RA, Wolf DE. 1995. Distribution and dynamics of mouse sperm surface galactosyltransferase: Implications for mammalian fertilization. Biochemistry 34:10027-10035.

Chamberlain LH. 2004. Detergents as tools for the purification and classification of lipid rafts. FEBS Lett 559:1-5.

Chamberlain LH, Burgoyne RD, Gould GW. 2001. SNARE proteins are highly enriched in lipid rafts in PC12 cells: Implications for the spatial control of exocytosis. Proc Natl Acad Sci USA 98:56195624.

Corselli J, Talbot P. 1987. In vitro penetration of hamster oocytecumulus complexes using physiological numbers of sperm. Dev Biol 122:227-242.

Cowan AE, Koppel DE, Vargas LA, Hunnicutt GR. 2001. Guinea pig fertilin exhibits restricted lateral mobility in epididymal sperm and becomes freely diffusing during capacitation. Dev Biol 236:502-509.

Cross NL. 2004. Reorganization of lipid rafts during capacitation of human sperm. Biol Reprod 71:1367-1373.

Delgado-Buenrostro NL, Hernandez-Gonzalez EO, Segura-Nieto M, Mujica A. 2005. Actin polymerization in the equatorial and postacrosomal regions of guinea pig spermatozoa during the acrosome reaction is regulated by G proteins. Mol Reprod Dev 70:198-210.

Fielding CJ, Fielding PE. 2004. Membrane cholesterol and the regulation of signal transduction. Biochem Soc Trans 32:65-69.

Flesch FM, Brouwers JF, Nievelstein PF, Verkleij AJ, van Golde LM, Colenbrander B, Gadella BM. 2001. Bicarbonate stimulated phospholipid scrambling induces cholesterol redistribution and enables cholesterol depletion in the sperm plasma membrane. J Cell Sci 114:3543-3555.

Foster LJ, De Hoog CL, Mann M. 2003. Unbiased quantitative proteomics of lipid rafts reveals high specificity for signaling factors. Proc Natl Acad Sci USA 100:5813-5818.

Gadella BM, Harrison RA. 2002. Capacitation induces cyclic adenosine $3^{\prime}, 5^{\prime}$-monophosphate-dependent, but apoptosis-unrelated, exposure of aminophospholipids at the apical head plasma membrane of boar sperm cells. Biol Reprod 67:340-350.

Golub T, Wacha S, Caroni P. 2004. Spatial and temporal control of signaling through lipid rafts. Curr Opin Neurobiol 14:542550 .

Hoessli DC, Ilangumaran S, Soltermann A, Robinson PJ, Borisch B, Nasir-Ud-Din. 2000. Signaling through sphingolipid microdomains of the plasma membrane: The concept of signaling platform. Glycoconj J 17:191-197.

Jaiswal BS, Eisenbach M. 2002. Capacitation. In: Hardy DM, editor. Fertilization. San Diego: Academic Press. pp 57-117.

Jones R, Shalgi R, Hoyland J, Phillips DM. 1990. Topographical rearrangement of a plasma membrane antigen during capacitation of rat spermatozoa in vitro. Dev Biol 139:349-362.

Madore N, Smith KL, Graham CH, Jen A, Brady K, Hall S, Morris R. 1999. Functionally different GPI proteins are organized in different domains on the neuronal surface. EMBO J 18:6917-6926.
Maier B, Medrano S, Sleight SB, Visconti PE, Scrable H. 2003. Developmental association of the synaptic activity-regulated protein arc with the mouse acrosomal organelle and the sperm tail. Biol Reprod 68:67-76.

Oh P, Schnitzer JE. 2001. Segregation of heterotrimeric G proteins in cell surface microdomains. G(q) binds caveolin to concentrate in caveolae, whereas G(i) and G(s) target lipid rafts by default. Mol Biol Cell 12:685-698.

Otter T, King SM, Witman GB. 1988. A two-step procedure for efficient electrotransfer of both high-molecular-weight (greater than 400,000) and low-molecular weight (less than 20,000) proteins. Anal Biochem 162:370-377.

Peterson RN, Russell LD. 1985. The mammalian spermatozoon: A model for the study of regional specificity in plasma membrane organization and function. Tissue Cell 17:769-791.

Prior IA, Harding A, Yan J, Sluimer J, Parton RG, Hancock JF. 2001. GTP-dependent segregation of H-ras from lipid rafts is required for biological activity. Nat Cell Biol 3:368-375.

Salaun C, James DJ, Chamberlain LH. 2004. Lipid rafts and the regulation of exocytosis. Traffic 5:255-264.

Salaun C, Gould GW, Chamberlain LH. 2005. The SNARE proteins SNAP-25 and SNAP-23 display different affinities for lipid rafts in PC12 cells: Regulation by distinct cysteine-rich domains. J Biol Chem 280:1236-1240.

Saxena N, Peterson RN, Sharif S, Saxena NK, Russell LD. 1986. Changes in the organization of surface antigens during in-vitro capacitation of boar spermatozoa as detected by monoclonal antibodies. J Reprod Fertil 178:601-614.

Schnitzer JE, Liu J, Oh P. 1995a. Endothelial caveolae have the molecular transport machinery for vesicle budding, docking, and fusion including VAMP, NSF, SNAP, annexins, and GTPases. J Biol Chem 270:14399-14404.

Schnitzer JE, McIntosh DP, Dvorak AM, Liu J, Oh P. 1995b. Separation of caveolae from associated microdomains of GPI-anchored proteins. Science 269:1435-1439.

Selvaraj V, Asano A, Buttke DE, McElwee JL, Nelson JL, Wolff CA Merdiushev T, Fornes MW, Cohen AW, Lisanti MP, Rothblat GH, Kopf GS, Travis AJ. 2006. Segregation of micron-scale membrane sub-domains in live murine sperm. J Cell Physiol 206:636-646.

Shadan S, James PS, Howes EA, Jones R. 2004. Cholesterol efflux alters lipid raft stability and distribution during capacitation of boar spermatozoa. Biol Reprod 71:253-265.

Sleight SB, Miranda PV, Plaskett NW, Maier B, Lysiak J, Scrable H, Herr JC, Visconti PE. 2005. Isolation and proteomic analysis of mouse sperm detergent-resistant membrane fractions: Evidence for dissociation of lipid rafts during capacitation. Biol Reprod 2005.; 73:721-729.

Sowa G, Pypaert M, Sessa WC. 2001. Distinction between signaling mechanisms in lipid rafts vs. caveolae. Proc Natl Acad Sci USA 98:14072-14077.

Talbot P. 1985. Sperm penetration through oocyte investments in mammals. Am J Anat 174:331-346.

Travis AJ, Merdiushev T, Vargas LA, Jones BH, Purdon MA, Nipper RW, Galatioto J, Moss SB, Hunnicutt GR, Kopf GS. 2001. Expression and localization of caveolin-1, and the presence of membrane rafts, in mouse and Guinea pig spermatozoa. Dev Biol 240:599-610.

Trevino CL, Serrano CJ, Beltran C, Felix R, Darszon A. 2001 Identification of mouse trp homologs and lipid rafts from spermatogenic cells and sperm. FEBS Lett 509:119-125.

van Gestel RA, Brewis IA, Ashton PR, Helms JB, Brouwers JF, Gadella BM. 2005. Capacitation-dependent concentration of lipid rafts in the apical ridge head area of porcine sperm cells. Mol Hum Reprod 11:583-590.

Visconti PE, Bailey JL, Moore GD, Pan D, Olds-Clarke P, Kopf GS 1995a. Capacitation of mouse spermatozoa. I. Correlation between the capacitation state and protein tyrosine phosphorylation. Development 121:1129-1137.

Visconti PE, Moore GD, Bailey JL, Leclerc P, Connors SA, Pan D, OldsClarke P, Kopf GS. 1995b. Capacitation of mouse spermatozoa. II Protein tyrosine phosphorylation and capacitation are regulated by a cAMP-dependent pathway. Development 121:1139-1150.

Visconti PE, Olds-Clarke P, Moss SB, Kalab P, Travis AJ, de las Heras M, Kopf GS. 1996. Properties and localization of a tyrosine 
phosphorylated form of hexokinase in mouse sperm. Mol Reprod Dev 43:82-93.

Visconti PE, Galantino-Homer H, Ning X, Moore GD, Valenzuela JP, Jorgez CJ, Alvarez JG, Kopf GS. 1999a. Cholesterol efflux-mediated signal transduction in mammalian sperm. beta-cyclodextrins initiate transmembrane signaling leading to an increase in protein tyrosine phosphorylation and capacitation. J Biol Chem 274:3235-3242.
Visconti PE, Ning X, Fornes MW, Alvarez JG, Stein P, Connors SA, Kopf GS. 1999b. Cholesterol efflux-mediated signal transduction in mammalian sperm: Cholesterol release signals an increase in protein tyrosine phosphorylation during mouse sperm capacitation. Dev Biol 214:429-443.

Ward CR, Kopf GS. 1993. Molecular events mediating sperm activation. Dev Biol 158:9-34. 